\title{
How Clean is Your Capsule?
}

\author{
W. T. GREEN and D. L. BOASE \\ Portsmouth
}

\begin{abstract}
Summary
Proliferation of residual lens epithelial cells is believed to be the major cause of posterior capsule opacification following extracapsular cataract extraction. During surgery these cells can be visualised with appropriate illumination facilitating their mechanical removal with the McIntyre cannula. When flat preparations of the anterior capsule are examined by light microscopy, the areas 'cleaned' of cells in this way appear transparent but scanning electron microscopy reveals tufts of remaining debris which may represent points of cellular attachment to the capsule.

Control of lens epithelial cell proliferation is important for the future development of cataract surgery.
\end{abstract}

The undoubted advantages of extracapsular cataract extraction are offset in many patients by posterior capsule opacification requiring capsulotomy. Not only is this disappointing for the patient, but the procedure carries a risk of serious complications.

The major cause of posterior capsule opacification is proliferation of residual lens epithelial cells. ${ }^{1}$ If these cells could be removed at the time of surgery we believe that the incidence of posterior capsule opacification and the need for subsequent capsulotomy would be reduced. A study was therefore undertaken to determine whether these cells could be visualised and physically removed during surgery.

In one case an attempt was made to estimate the number of cells remaining after an extracapsular cataract extraction in a cadaver eye with a canopener capsulotomy.

\section{Methods and Results}

Illumination and visualisation during surgery Intercapsular (endocapsular) cataract extraction was performed during cataract surgery and also on human cadaver eyes. A horizontal capsulotomy in the upper part of the lens allowed nucleus removal. Irrigation and aspiration of the cortical lens material was then carried out using a McIntyre cannula with Hartman's irrigation solution. During in vitro surgery this was aided by first removing the entire cornea and iris to improve visualisation and explore different methods of illumination.

The importance of illumination was first suspected when it was observed, during routine intercapsular cataract extraction, that the lens epithelial cells lining the inner aspect of the anterior capsule could be visualised much more clearly with the assistant microscope attachment (at $30^{\circ}$ of incidence) on a Zeiss operating microscope (OMPI 6) than by the operating surgeon with his coaxial view. We therefore investigated the use of oblique illumination using fibreoptic light sources with focusing lenses held by adjustable clamps to provide light from any angle.

It was found that oblique illumination during in vitro surgery allowed visualisation of details on the anterior capsule with the lens 

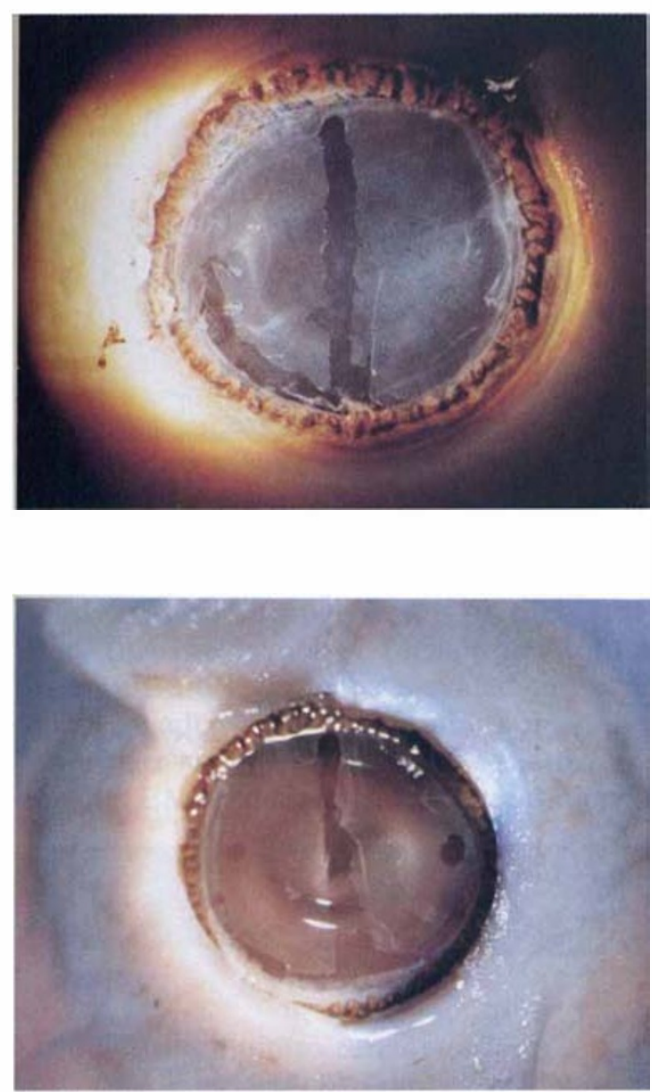

epithelial cells standing out more clearly against the dark background (Figs. 1,2,3) than with the red reflex provided by coaxial illumination (Figs.1a,2a,3a).

The best arrangement was found to be one fibreoptic light source on either side at an angle of $30^{\circ}$ to the horizontal with the light focused as much as possible onto the lens capsule (Fig. 4).

Having illuminated the lens epithelial cells in this way it was found that they could be removed mechanically by applying the port of the McIntyre cannula to the inner aspect of the anterior capsule and moving it gently from side to side while applying a gentle vacuum. However, cleaning cells from a free edge of capsule is extremely difficult due to loss of the vacuum (which keeps the port applied to the capsule) as the capsule edge is approached. Once learned during in vitro surgery, this technique can be applied to live intercapsular surgery, even though the iris (no matter how

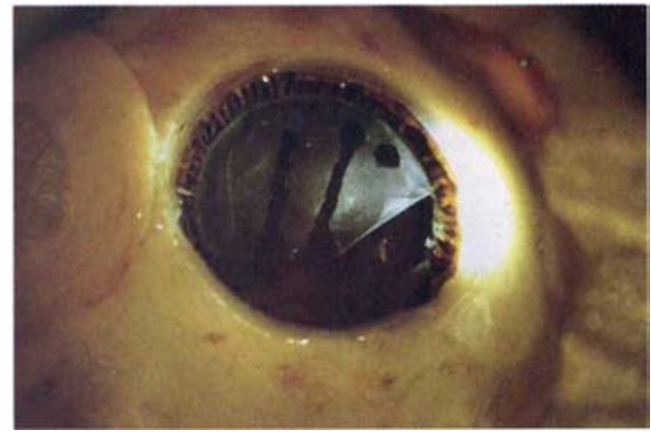

Figs. 1,2,3. In vitro surgery; cornea and iris removed demonstrating streaks of removed lens epithelial cells in each case with oblique (sixty degrees from the vertical) illumination.

well dilated the pupil) obscures details of the fornix of the capsule bag.

\section{Histology}

\section{Light Microscopy}

For the purposes of demonstrating histologically that it is indeed cell removal which is being witnessed, an area of anterior capsule was cleaned in cadaver eyes. The capsule bag was then stained with toludine blue (which stains cells but not 'cleaned' capsule) and the anterior capsule excised. The junction between cell laden, and 'cleaned' capsule was then examined by light microscopy at various magnifications.

These preparations showed clearly that the presumed areas of 'clean' capsule were indeed free of cells, and the capsule appeared transparent in these areas using this stain (Fig. 5).

\section{Scanning Electron Microscopy}

No suitable means was found to prevent the 

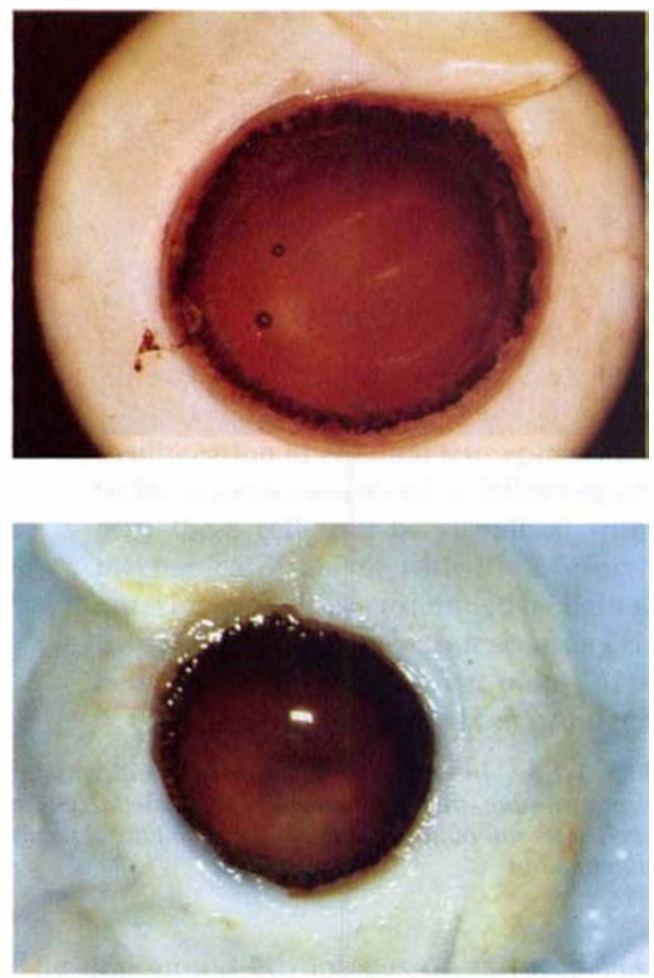

anterior capsule specimens from rolling up into a 'scroll' prior to EM since any substance introduced to hold the specimen flat could produce artefacts. A number of rolled up specimens were therefore studied and it was soon apparent that some had lens epithelial cells on the outside whereas others had rolled up with the cells inside and these latter specimens were difficult to study. Specimens were

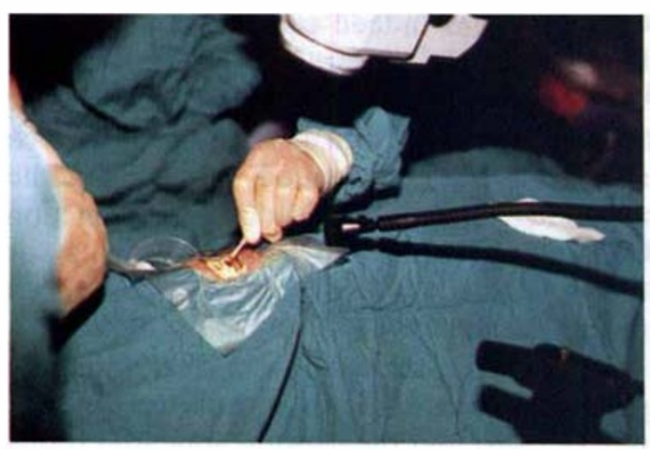

Fig. 4. Oblique illumination provided at sixty degrees from the vertical by an adjustable fibre-optic cable (covered with sterile plastic tubing during surgery). Second cable held with clamp (lower right).

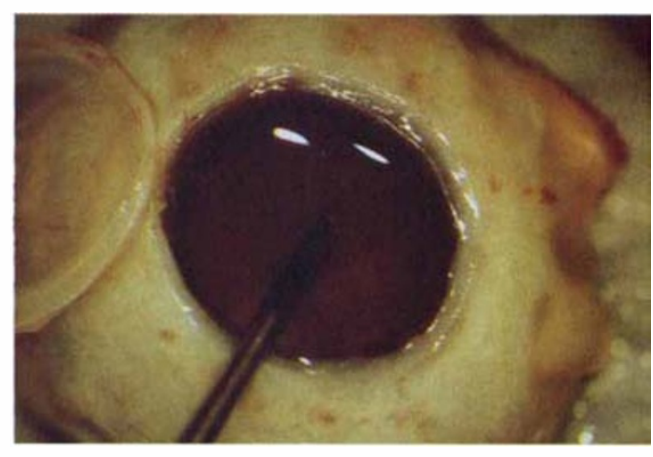

Figs. 1a,2a,3a. The same three cadaver eyes viewed with coaxial illumination.

placed in $2 \%$ aqueous osmium tetroxide at 4 degree $\mathrm{C}$ in the dark for 16 hours; allowed to attain room temperature and washed in distilled water; dehydrated through a $10 \%$ graded ethanol series and transferred to absolute ethanol for 30 minutes.

The specimens were then infiltrated with a series of increasing concentrations of acetone; dried at critical point (Polaron E3000 appara-

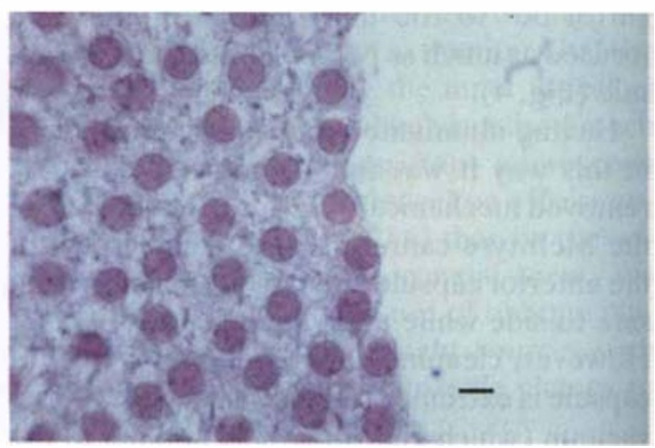

Fig. 5. Light micrograph showing junction between lens epithelial cells (left), and 'cleaned' anterior capusle (right), the latter being transparent with this stain (toludine blue), (Bar $=5 \mu)$. 
tus) using liquid carbon dioxide; mounted on stubs with double-sided adhesive and gold coated (25 nm thickness) in a Polaron 5000 Diode Sputtering system. The specimens were then examined in a JEOL $35 \mathrm{C}$ scanning electron microscope at a range of accelerating voltages.

Using this method, the surface of apparently 'cleaned' capsule was found to have small tufts of retained debris (Fig. 6). These tufts may represent firm attachments of the lens epithelial cells to the capsule, although it is interesting to note that these were not present in a ratio of one per cell (Fig. 7). An 'edge' view of these tufts was obtained by studying a region where a split in one specimen fortuitously passed through some of these tufts (Fig. 8). The layers of capsule adjacent to each tuft are seen to be elevated providing further evidence of cellular adhesion.

Further studies using scanning and transmission EM with serial sections are underway to further define these apparent areas of adhesion.

\section{Discussion}

Coaxial illumination is considered desirable for cataract surgery as it provides a bright red reflex which is especially helpful during aspiration of cortical lens material. However, methods of oblique illumination highlight details of the surgical anatomy and allow better visualisation of the lens epithelial cells which can then be removed by simple mechanical means.

The layer of lens epithelial cells probably reflects light by specular reffection with the arrangement of light described here, allowing the cells to be clearly seen àgainst what is now a black background. With recent concern about incident light upon the macula reaching phototoxic levels during surgery, this technique offers the advantage of reduced direct illumination of the macula.

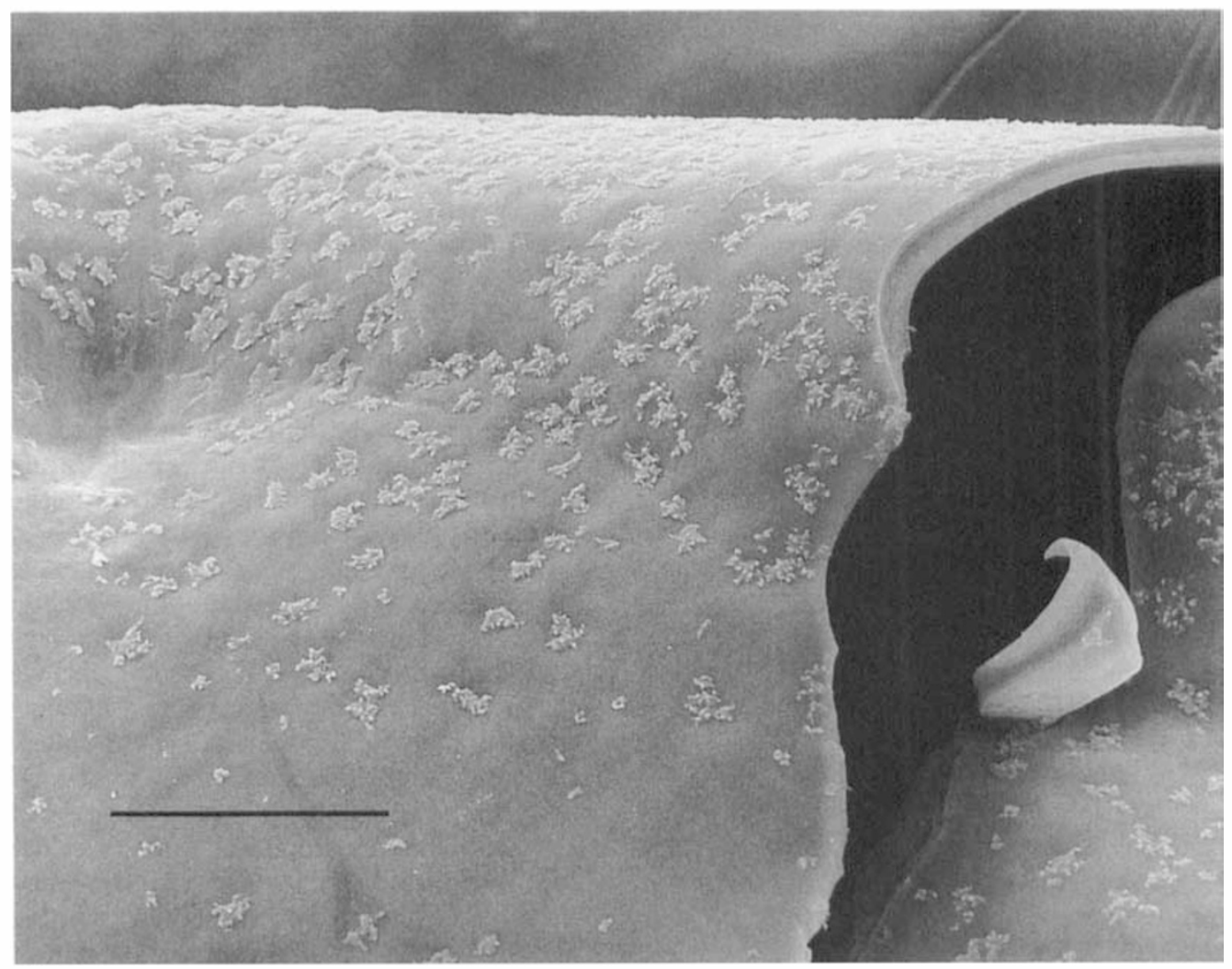

Fig. 6. Scanning electron micrograph showing the 'inner' surface of one anterior capsule specimen previously 'cleaned' of cells using the McIntyre cannula. Patches of debris remain attached to the capsule. (Bar=100u). 


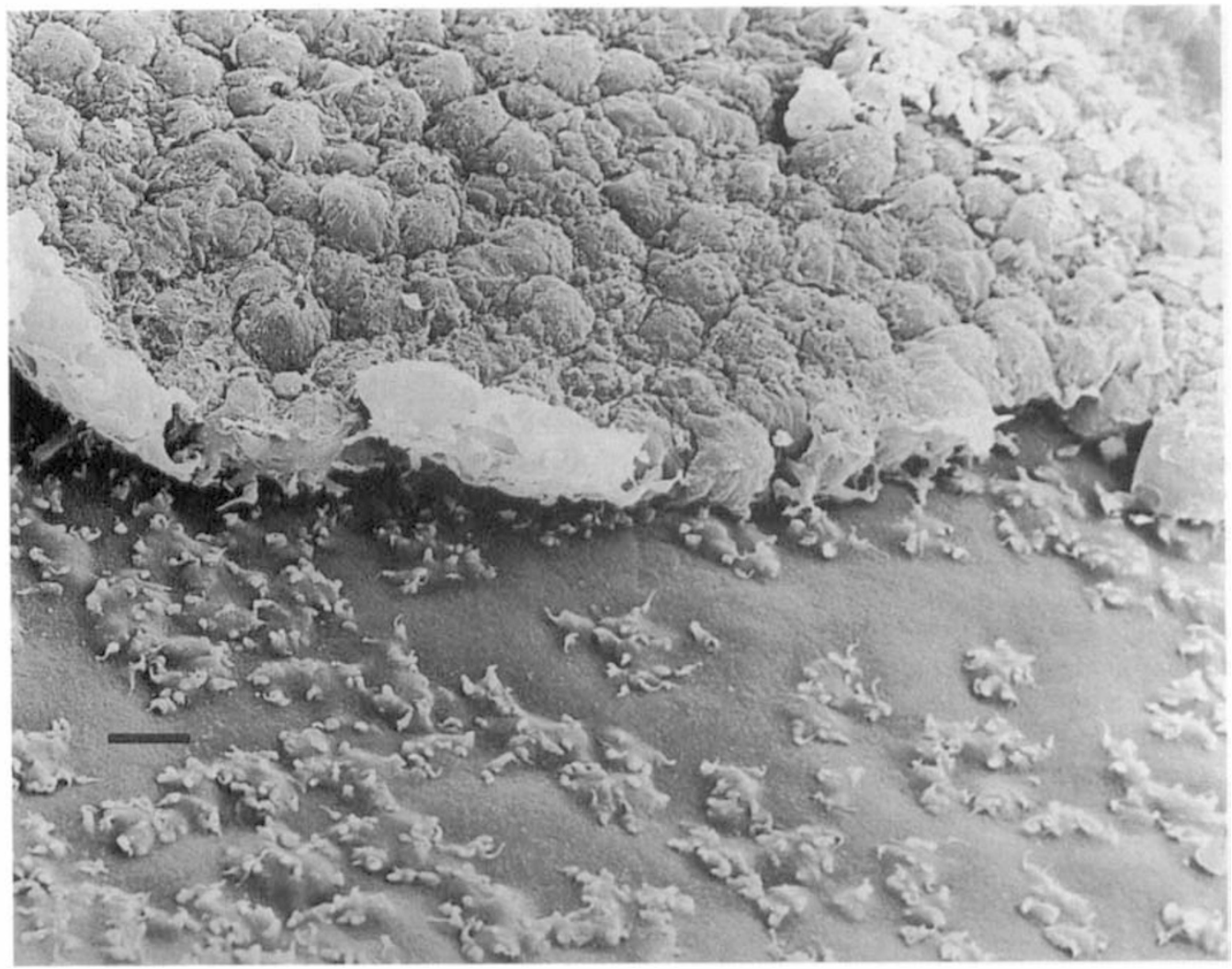

Fig. 7. Scanning electron micrograph of junction between cells (above) and 'cleaned' capsule (below) with retained debris. (Bar $=10 \mathrm{u})$.

Attention is frequently directed to cleaning the posterior capsule during cataract surgery, and the lens epithelial cells ignored. This paper describes a method of visualising the lens epithelial cells during in vitro surgery and their mechanical removal. These methods have been applied to live intercapsular surgery and the equipment used to produce oblique illumination for this study was found to provide excellent visualisation, but would require further refinements to be practical in the context of a sterile operating field. Ideally, one should have the ability to vary the direction of one or more sources of illumination during surgery to suit the needs of the moment, and newly available microscopes with a range of fibreoptic attachments to various light sources will hopefully provide this.

This method of lens epithelial cell removal is not practical with a 'canopener' capsulo- tomy (for the reasons given above). It is sometimes erroneously assumed that a wide capsulotomy of this type will remove all the cells. Unfortunately the cells extend into the far recesses ('equator') of the capsule fornix and in cataractous lenses frequently onto the peripheral posterior capsule. ${ }^{2}$ Therefore even the widest surgical capsulotomy would leave an extensive area of lens epithelium.

To demonstrate the extent of residual lens epithelium with this type of capsulotomy, we examined a capsule bag preparation following the widest possible canopener capsulotomy in one cadaver eye. The specimen was stained with toludine blue, carefully dissected free of all attachments and radial cuts made in the anterior capsule (Figs. 9,10) so that the specimen could be laid flat on a Neubauer counting chamber (consisting of a slide with a grid of known dimensions marked on its surface), and the total number of remaining lens epi- 


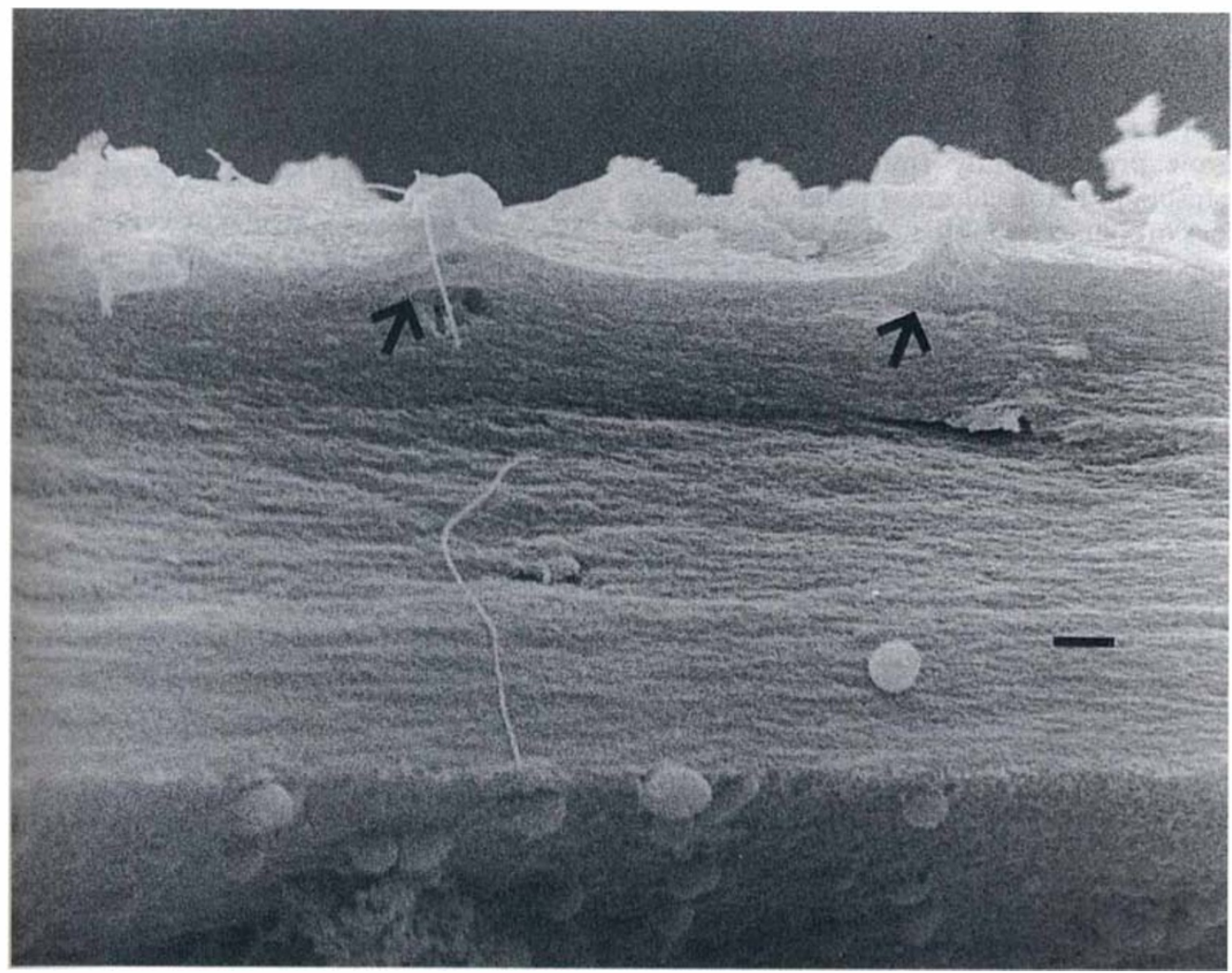

Fig. 8. Scanning electron micrograph view of anterior capsule edge formed by an artefactual split (seen in Fig. 6) which has fortuitously passed through retained 'tufts' on the capsule surface from which cells have been mechanically removed. $($ Bar $=1 \mu)$.

thelial cells estimated. ${ }^{3}$ In this case the number of remaining cells was found to be approxiamtely 100,000 (4000 per square mm with a 25 square $\mathrm{mm}$ total stained area of capsule).

This represents a large pool of potentially proliferating lens epithelial cells living in a natural culture medium. Surgical capsulo-

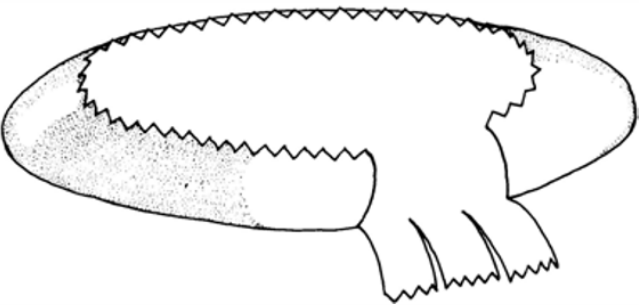

Fig. 9. Diagram to show the excised capsule bag in which radial incisions are made in the anterior capsule 'frill' which remains following a canopener capsulotomy in a cadaver eye. tomy for cataract extraction would normally be smaller than in this case and with recent trends towards leaving larger anterior capsule

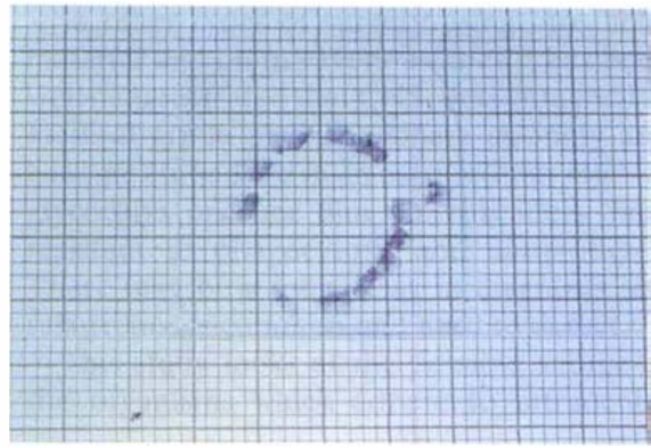

Fig. 10. Photograph of specimen prepared as in Fig. 9 and stained with toludine blue (which stains cells but not the central cell-free posterior capsule). The small squares of the grid are $1 \mathrm{~mm}^{2}$. 
flaps in intercapsular surgery, still greater numbers of lens epithelial cells must remain.

It has been shown that lens epithelial cells grow predominantly from a cut edge of capsule in tissue culture, presumably due to lack of contact inhibition. ${ }^{4}$ It may therefore be important to remove lens epithelial cells from the area of capsule through which one will subsequently perform the capsulotomy and capsulorrhexis during intercapsular cataract surgery (to leave a 'cell free' capsule edge), particularly since relatively large capsule flaps often remian following this surgery. Whether this is of clinical benefit in reducing the incidence of posterior capsule opacification is the subject of further investigation.

\section{References}

${ }^{1}$ Green WR and McDonnell PJ: Opacification of the posterior capsule. Trans Ophthalmol Soc UK 1985, 104: 727-39.

${ }^{2}$ Marshall J, Beaconsfield M, Rothery S: The anatomy and development of the human lens and zonules. Trans Ophthalmol Soc UK 1982, 102: 423-40.

${ }^{3}$ Dacie JV and Lewis SM, eds: Counting red blood cells with a Neubauer counting chamber In: Practical Haematology, 3rd ed., Churchill, London 1963, 18-27.

${ }^{4}$ Jacob TJC, Humphrey RC, Davies EG, Thompson GM: Cytological factors relating to posterior capsule opacification following cataract surgery. $\mathrm{BrJ}$ Ophthalmol 1987, 71: 659-63. 